\title{
Comparative Evaluation of Marginal Fit of Three Provisional Restorative Materials in Different Mediums: An In-Vitro Study
}

\author{
Manju Choudhary \\ MDS, (Prosthodontics), Asst. Professor, Dept. of Dentistry, K.M Medical College \& Hospital, Mathura. (UP)
}

\begin{abstract}
Temporaries are used as placeholders before the permanent crowns are installed. If the temporary crown is not fitted properly the tooth can be subject to increased decay and gums can become inflamed causing gingivitis which leads to other more serious problems. A provisional fixed restoration will provide a template for defining tooth contour, esthetics, proximal contacts, ridge contacts and occlusion. Margins made by the indirect technique are considered to be more accurate than those made by the direct technique. The purpose of this in vitro study was to compare the marginal accuracy of provisional crowns made from three different biomaterials using the established indirect method in different environments and to evaluate the effect of water absorption on polymerization shrinkage and the effect of polymerization shrinkage occurring in dry storage for a week.
\end{abstract}

Method: Variables used in this study are: a) Revotek LC-light cured composite b) Protemp II c) poly methyl methacrylate. An aluminium master die was machined with dimensions: $5 \mathrm{~mm}$ length, $10 \mathrm{~mm}$ gingival diameter, 5 degree taper, and $1 \mathrm{~mm}$ shoulder then dental stone die was prepared by making an impression of this experimental model using a poly vinyl siloxane material. Both group A and group B consisted of seven specimens each of DPI, ProtempII, Revotek LC. The specimens were evaluated using measuring microscope (Biolux), which had an eyepiece graticule of 1/10th of an mm. The seven specimens in group A (DPI, ProtempII, Revotek LC) were kept in air at room temperature for one week on the dental stone cast and the readings were tabulated and the mean values were obtained. The seven specimens in group B (DPI, ProtempII, and Revotek LC) were kept in water at room temperature for one week and the readings were tabulated in the same manner and the mean values were obtained.

Results: Comparative statistics of the mean values of the specimens kept in air at room temperature showed that DPI had the least marginal discrepancies followed by ProtempII, Revotek LC. The values were highly significant (.002) Comparative statistics of the mean values of the specimens kept in water at room temperature showed that DPI had the least marginal discrepancies followed by Revotek LC, ProtempII. The values were highly significant (.009).

Conclusion: After one week in air at room temperature and after one week in water at room temperature DPI recorded the minimal marginal discrepancy. When stored in air at room temperature, DPI had the best marginal adaptation. All of the materials showed evidence of continued polymerization shrinkage after storage in air for a week. Water absorption compensated for polymerization shrinkage in DPI and ProtempII whereas Revotek LC was an exception.

Keywords: light cured composite, Protemp IIBis-acryl composite, poly methyl methacrylate, marginal adaptation, provisional restoration

\section{INTRODUCTION}

Any dental procedure or implantation has the potential to be uncomfortable for the patient. Sometimes the period between the initial consultation visit and the final procedure that results in the dentist placing permanent crowns can span months. Creating custom crowns for patients is an important process. Temporaries are used as placeholders before 
the permanent crowns are installed. Sometimes patients have to wear the temporary crowns for months between their visits. If the temporary crown is not fitted properly the tooth can be subject to increased decay and gums can become inflamed causing gingivitis which leads to other more serious problems. There are multiple areas of critical concern with provisional restorations including esthetics, comfort, speech and function, periodontal health, maxillomandibular relationships, and continued evaluation of the fixed prosthodontic treatment plan. [1-5] Biologically acceptable fixed prosthodontic treatment demands that prepared teeth be protected and stabilized with provisional restorations that resemble the form and function of the planned definitive treatment. [6] They assist in the maintenance of periodontal health ${ }^{[7]}$ and promote guided tissue healing by providing a matrix for surrounding gingival tissues ${ }^{[8]}$. A provisional fixed restoration will provide a template for defining tooth contour, esthetics, proximal contacts, ridge contacts and occlusion [9] and for evaluating the potential consequences from an alteration in the vertical dimension of occlusion. The ideal properties of the provisional restorative material are good marginal adaptation; adequate retention and resistance to dislodgment during normal masticatory function; strong, durable, and hard; nonirritating to pulp and other tissues; low exothermic reaction; nonporous and dimensionally stable; esthetically acceptable shade selection, finishes to a highly polished, plaque- and stain-resistant surface. [10] The indirect technique has been associated with superior fit and pulpal protection. [11-12] Margins made by the indirect technique are considered to be more accurate than those made by the direct technique. ${ }^{[11-13]}$ The purpose of this in vitro study was to compare the marginal accuracy of provisional crowns made from three different biomaterials using the established indirect method in different environments.

\section{OBJECTIVES}

The objectives of this study are

1) To evaluate the marginal accuracy of three different resins by using indirect technique.

2) To evaluate the effect of water absorption on polymerization shrinkage and the effect of polymerization shrinkage occurring in dry storage for a week.

\section{METHODOLOGY}

Variables used in this study are: a) Revotek LC-light cured composite (GC Corp) b) Protemp II-Bis -acryl composite (3M-ESPE) c) Conventional poly methyl methacrylate in powder and liquid form (DPI)

Specimen fabrication: metal die preparation: An aluminium master die was machined to the following dimensions: $5 \mathrm{~mm}$ length, $10 \mathrm{~mm}$ gingival diameter, 5 degree taper, and $1 \mathrm{~mm}$ shoulder. A flat surface machined at a 30 degree angle to the long axis was made on one side of the die to help standardize the crown placement. Two points of reference were placed on the facial and lingual gingival finish lines so that the measurements made were consistent. The experimental model, simulating the clinical situation before and after tooth preparation, consisted of three metal dies with the master die in the middle. A full crown was placed over the master die to simulate the unprepared tooth. No measurable gaps were observed at the margin of the crown. Preparation of dental stone die: An impression of this experimental model was made using a poly vinyl siloxane material (Aquasil-ultra LV, Dentsply DeTrey) in a custom made box. Dental stone was poured into the impression. A stone model, simulating the tooth before preparation, was obtained from this impression. The crown was then removed from the metal die. Three impressions of the experimental model were made in the same manner with the same impression material. After the impression was retrieved, dental stone was poured into the three impressions. A total of 42 dies 
were made with dental stone. Fabrication of mold Group 1 (DPI) The fourteen stone dies were flasked and dewaxed following the standard procedure. The monomer: polymer ratio used was $1: 3$ by volume. Fourteen provisional resin specimens were obtained in the same manner. Group 2(PROTEMP-II) The two pastes are mixed according to manufactures instruction on a paper pad for $30-45 \mathrm{sec}$.The stent was lubricated with petrolatum, for the ease of removal. The material was then loaded into the stent and the stent was seated on to the stone die. After a setting time of $4 \mathrm{~min}$ and $30 \mathrm{sec}$ Protemp was removed from the stone die. It was bench cured for 10 minutes. Fourteen provisional resin specimens were obtained in the same manner. Group 3(REVOTEK LC) To fabricate the restoration, a small portion of the material was cut from the stick using the plastic instrument supplied along with the pack and adapted to the stone die. Then the stone die along with resin composite and the stent was placed in to the light-curing unit (labolight -II) for 60 seconds. Fourteen provisional resin specimens were obtained in the same manner. After the fabrication of fourteen provisional resin specimens each (DPI, ProtempII, Revotek LC) using indirect technique, they were divided into two groups A and B. Both group A and group B consisted of seven specimens each of DPI, ProtempII, Revotek LC. The specimens were evaluated using measuring microscope (Biolux), which had an eyepiece graticule of $1 / 10$ th of an $\mathrm{mm}$. The readings were tabulated under group A and group B of both buccal and lingual reference points. Then the mean values of buccal and lingual reference points were obtained of each specimen. The values were then multiplied with 18 (magnification factor) for obtaining the values in $\mu \mathrm{mm}$. The seven specimens in group A (DPI, ProtempII, Revotek LC) were kept in air at room temperature for one week on the dental stone cast and the readings were tabulated in the same manner and the mean values were obtained. The seven specimens in group B (DPI, ProtempII, and Revotek LC) were kept in water at room temperature for one week and the readings were tabulated in the same manner and the mean values were obtained.

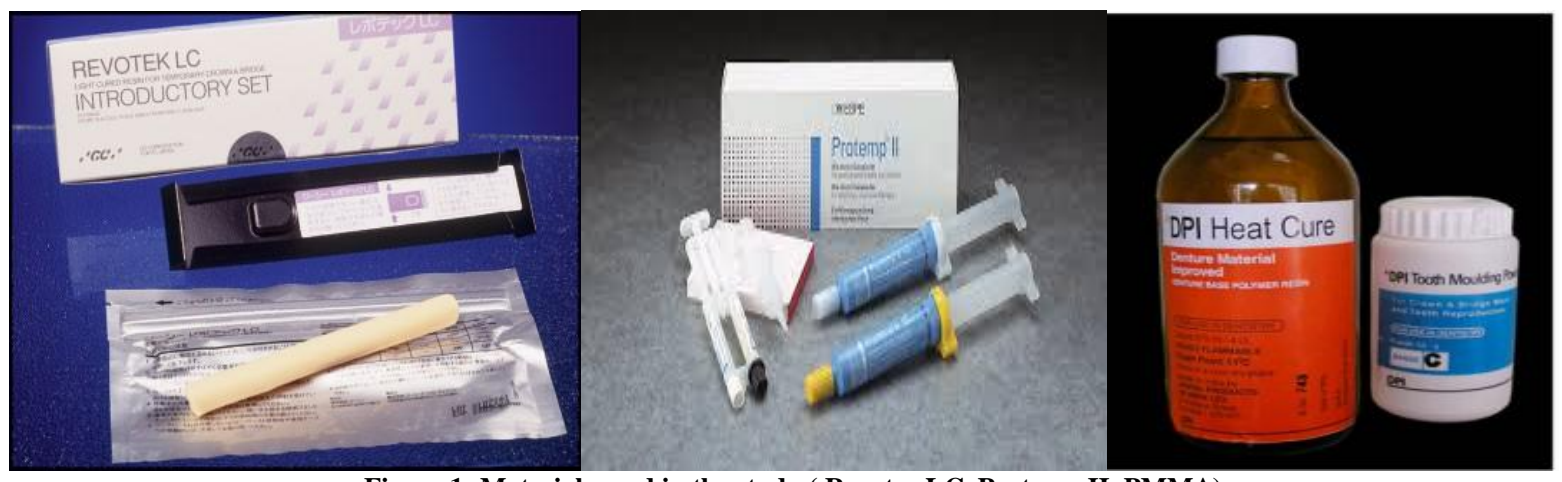

Figure 1: Materials used in the study ( Revotec LC, Protemp II, PMMA)

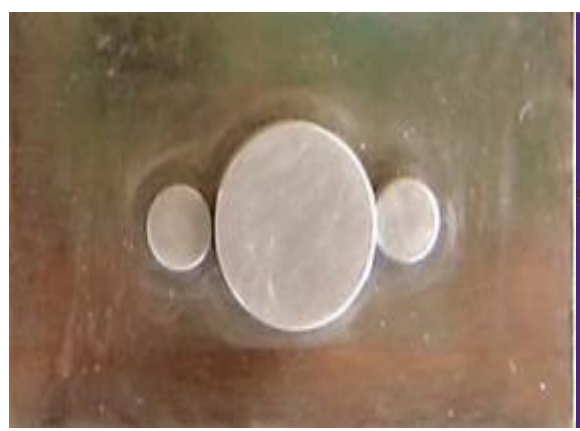

Figure 2: Metal die

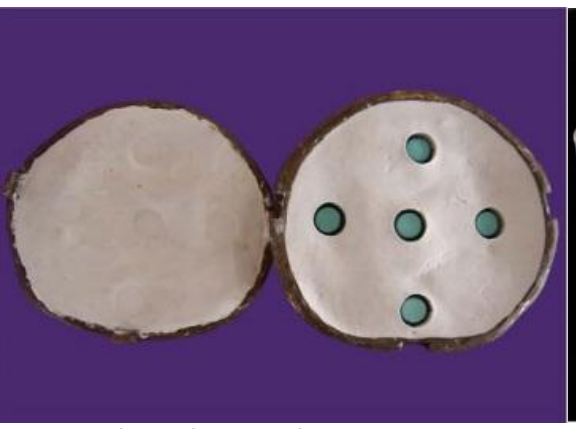

Figure 3: Dewaxing

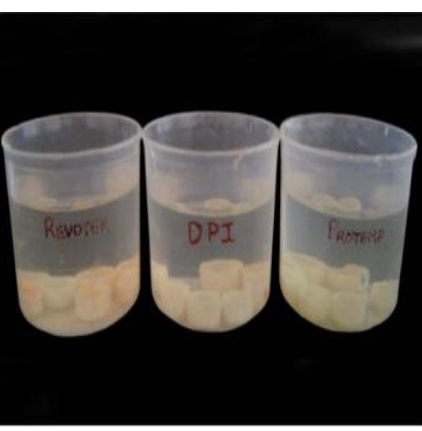

Figure 4: Wet specimens 


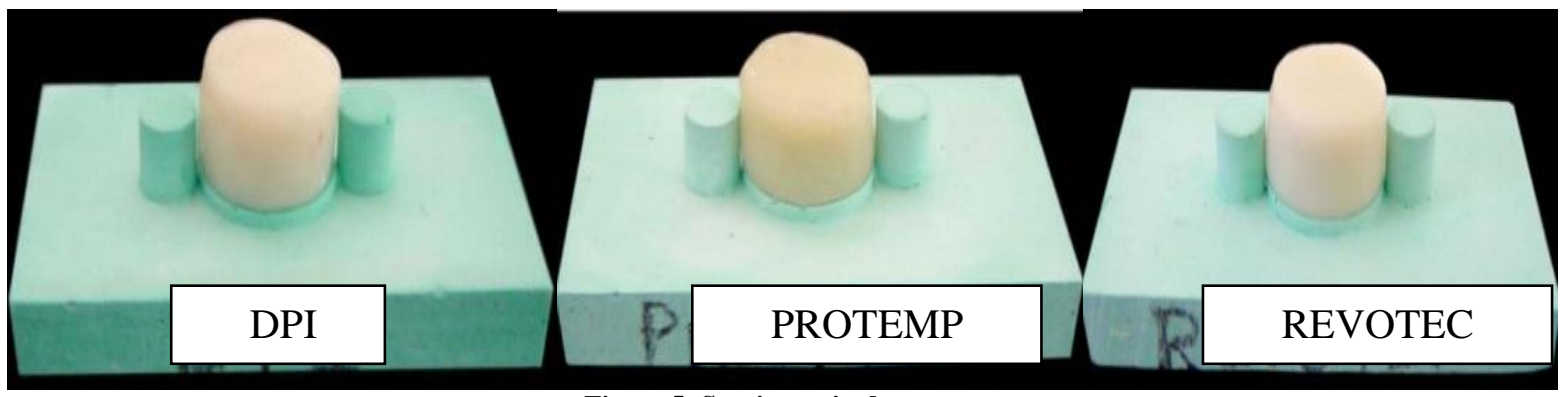

Figure 5: Specimens in dry storage

\section{RESULTS}

Table 1 : Master table showing comparison of marginal discrepancies of the specimens after one week in water at room temperature

\begin{tabular}{|l|c|c|c|c|c|c|c|c|c|}
\hline Specimen & \multicolumn{3}{|c|}{ REVOTEK LC (Group I) } & \multicolumn{3}{l|}{ PROTEMP (Group II) } & \multicolumn{2}{l|}{ DPI (Group III) } \\
\hline & Buccal & Lingual & Mean & Buccal & Lingual & Mean & Buccal & Lingual & Mean \\
\hline & $\mu \mathrm{mm}$ & $\mu \mathrm{mm}$ & $\mu \mathrm{mm}$ & $\mu \mathrm{mm}$ & $\mu \mathrm{mm}$ & $\mu \mathrm{mm}$ & $\mu \mathrm{mm}$ & $\mu \mathrm{mm}$ & $\mu \mathrm{mm}$ \\
\hline 1 & 54 & 90 & 72 & 108 & 144 & 126 & 54 & 90 & 72 \\
\hline 2 & 90 & 90 & 90 & 90 & 108 & 99 & 90 & 90 & 90 \\
\hline 3 & 72 & 90 & 81 & 72 & 108 & 90 & 72 & 90 & 81 \\
\hline 4 & 90 & 72 & 81 & 54 & 72 & 63 & 90 & 72 & 81 \\
\hline 5 & 144 & 108 & 126 & 108 & 72 & 90 & 144 & 108 & 126 \\
\hline 6 & 72 & 108 & 90 & 162 & 144 & 153 & 72 & 108 & 90 \\
\hline 7 & 108 & 108 & 108 & 72 & 144 & 108 & 108 & 108 & 108 \\
\hline
\end{tabular}

Table 2 : Master table showing comparison of marginal discrepancies of the specimens after one week in air at room temperature.

\begin{tabular}{|l|c|c|c|c|c|c|c|c|c|}
\hline Specimen & REVOTEK LC (Group I) & \multicolumn{2}{|c|}{ PROTEMP (Group II) } & \multicolumn{3}{|c|}{ DPI (Group III) } \\
\hline & Buccal & Lingual & Mean & Buccal & Lingual & Mean & Buccal & Lingual & Mean \\
\hline & $\mu \mathrm{mm}$ & $\mu \mathrm{mm}$ & $\mu \mathrm{mm}$ & $\mu \mathrm{mm}$ & $\mu \mathrm{mm}$ & $\mu \mathrm{mm}$ & $\mu \mathrm{mm}$ & $\mu \mathrm{mm}$ & $\mu \mathrm{mm}$ \\
\hline 1 & 108 & 144 & 126 & 108 & 72 & 90 & 72 & 144 & 108 \\
\hline 3 & 72 & 144 & 108 & 126 & 108 & 117 & 144 & 90 & 117 \\
\hline 4 & 144 & 90 & 117 & 90 & 162 & 126 & 72 & 108 & 90 \\
\hline 5 & 108 & 144 & 126 & 144 & 162 & 153 & 108 & 144 & 126 \\
\hline 6 & 162 & 126 & 144 & 108 & 72 & 90 & 72 & 72 & 72 \\
\hline 7 & 108 & 162 & 135 & 72 & 72 & 72 & 126 & 72 & 99 \\
\hline
\end{tabular}

Table VI: Comparative statistics of the mean values of the different brand specimens kept in water at room temperature and specimens kept in air at room temperature

\begin{tabular}{|c|c|c|c|c|c|c|}
\hline Medium & Brand & $\mathbf{N}$ & Mean & Standard Deviation & $\mathbf{H}$ & p \\
\hline \multirow[t]{3}{*}{ Air } & Protemp & 7 & 116.7143 & 8.5579 & \multirow{3}{*}{12.59} & \multirow{3}{*}{$.002 \mathrm{hs}$} \\
\hline & Revotek LC & 7 & 122.8571 & 8.4148 & & \\
\hline & DPI & 7 & 100.0000 & 6.0277 & & \\
\hline \multirow[b]{3}{*}{ Water } & Protemp & 7 & 104.1429 & 15.2690 & \multirow{3}{*}{9.42} & \multirow{3}{*}{$.009 \mathrm{hs}$} \\
\hline & Revotek LC & 7 & 94.0000 & 17.8606 & & \\
\hline & DPI & 7 & 76.7143 & 13.1747 & & \\
\hline
\end{tabular}

$H=$ Kruskal - Wallis test $p>0.05$-Non- significant $p<0.05$ - Significant $p<.01$ highly significant $p<.001$ very highly significant

Comparative statistics of the mean values of the specimens kept in air at room temperature showed that DPI had the least marginal discrepancies followed by ProtempII, Revotek LC. The values were highly significant (.002). So DPI is considered to be having superior marginal adaptation of the specimens kept in air at room temperature among the three groups.

Comparative statistics of the mean values of the specimens kept in water at room temperature showed that DPI had the least marginal discrepancies followed by Revotek LC, ProtempII. The values were highly significant (.009). So DPI is considered to be having superior marginal adaptation of the specimens kept in water at room temperature among the three groups.

\section{DISCUSSION}

An increase in the size of marginal gap is of clinical significance because they facilitate plaque accumulation, which leads to gingival inflammation and ulceration of the inner wall of the gingival crevice. Greater marginal gaps results in increased surface area and thus permit rapid dissolution of temporary luting cements and 
accumulation of debris in the resulting void at the margin of tooth crown interface. Poly methyl methacrylate resin possesses satisfactory overall physical properties, including marginal finish and its potential to impart and maintain polish and fine marginal adaptation have been reported. ${ }^{[14-}$ 16] Bis-acryl composite is available in its convenient, cartridge-based dispensing system, results in a more accurately proportioned and consistent mix and has a low exothermic reaction. However, the material breaks when placed area of moderate stress. Visible light cure composite resin has gained popularity because of its ease of manipulation, and lack of exothermic reaction. Putty like consistency makes them easy to use. Composite resin can be finished and polished to an excellent level. However, the material appears to suffer from inherent brittleness. The marginal discrepancy of resin crowns using the direct technique is considered to be caused in part by improper seating of the crown after polymerization. ${ }^{[17]}$ A number of studies have focused on the effects of thermocycling on provisional crown margins. Takahiro ${ }^{[18]}$ et al reported that marginal gap changes were greater after hot thermocycling than cold thermocycling. Jerald Blum ${ }^{[19]}$ et al stated that improved marginal accuracy of poly methyl methacrylate provisional restorations occurred when a shoulder finish line was used compared with a chamfer marginal design. Dubois RJ ${ }^{[20]}$ et al has shown that light-polymerized materials provided significantly improved marginal accuracy relative to poly methyl methacrylate resin after thermocycling. According to Lepe X et al the marginal fit of composite provisional restoration is superior to that of poly methyl methacrylate in the initial stage. Due to the amount of polymerization shrinkage, provisional restorations made with poly methyl methacrylate resin have a tighter fit than composite in the later stages. It has been reported that provisional restorations were more accurate when an indirect method was used compared to a direct method. ${ }^{[11,12]}$, Koumjian and Holmes stated that marginal discrepancy increased after one week of storage in air, whereas water storage improved marginal adaptation for certain biomaterials.

\section{SUMMARY AND CONCLUSION}

Based on the observations of this study, the following conclusions were drawn

1. After one week in air at room temperature and after one week in water at room temperature DPI recorded the minimal marginal discrepancy. When stored in air at room temperature, DPI had the best marginal adaptation.

2. Although marginal gap of ProtempII was high after one hour after fabrication, it showed minimal variation when kept in dry and wet storage

3. All of the materials showed evidence of continued polymerization shrinkage after storage in air for a week.

4. Water absorption compensated for polymerization shrinkage in DPI and ProtempII whereas Revotek LC was an exception.

\section{REFERENCES}

1. Kaiser DA, Cavazos E Jr. Temporization techniques in fixed Prosthodontics. Dent Clin North Am 1985; 29:403-412.

2. Higginbottom FL. Quality provisional restorations: a must for successful restorative dentistry. Compend Contin Educ Dent 1995; 16:442-444. -7

3. Federick DR. The provisional fixed partial denture. J Prosthet Dent 1975; 34:520-526.

4. Shavell HM. Mastering the art of provisionalization. J Calif Dent Assoc 1979; 7:42-49.

5. Zinner ID, Small SA, Panno FV. Presurgical prosthetics and surgical templates. Dent Clin North Am 1989; 33:619-633.

6. Fox CW, Abrams BL, Doukoudakis A. Provisional restorations for altered occlusions. J Prosthet Dent 1984; 52:567572.

7. Vahidi F. The provisional restoration. Dent Clin North Am 1987;31:363-381 
8. Lowe RA. The art and science of provisionalization. Int $\mathrm{J}$ Periodontics Restorative Dent 1987; 7:64-73.

9. Zinner ID, Trachtenberg DI, Miller RD. Provisional restorations in fixed partial prosthodontics. Dent Clin North Am 1989; 33:355-377

10. David R. Burns, David A. Beck, Steven K. Nelson. A review of selected dental literature on contemporary provisional fixed prosthodontic treatment: Report of the Committee on Research in Fixed Prosthodontics of the Academy of Fixed Prosthodontics. J Prosthet Dent; 2003 ; 90 ;474-497

11. Crispin BJ, Watson JF, Caputo AA. The marginal accuracy of treatment restoration: a comparative analysis. J Prosthet Dent 1980; 44; 283-90.

12. Monday JJ, Blais D. Marginal adaptation of provisional acrylic resin. J Prosthet Dent $1985 ; 54 ; 194-7$.

13. Davidson CL, Feilzer AJ. Polymerization shrinkage and Polymerization shrinkage stress in polymer based restoratives. J Dent 1997; 25; 435-40.

14. Bargi N, Simmons EW. The marginal integrity of the temporary acrylic resin crown. J Prosthet Dent 1976; 36; 274-7.

15. Robinson FB, Hovijitra S. Marginal fit of direct temporary crowns. J Prosthet Dent $1982 ; 47 ; 390-2$.
16. Wang RL, Moore BK, Goodacre CJ .A comparison of resins for fabricating provisional fixed restoration. Int $\mathbf{J}$ Prosthodont 1989; 2; 173-84.

17. Stephen F Rosenstiel, Martin F Land, Junhei Fujimoto. Contemporary fixed prosthodontics. 3rd edition,2001; Mosby, 380-415.

18. Ogawa T, Aizawa S, Tanaka M, Matsuya S, Hasegawa A, Koyano K: Effect of water temperature on the fit of provisional crown margins during polymerization. J Prosthet Dent. 1999 Dec; 82(6):658-61

19. Blum J, Weiner S, Berendsen P. Effect of thermocycling on the margins of transitional acrylic resin crowns. J Prosthet Dent 1991; $65 ; 642-6$

20. Dubois RJ, Kyriakakis P, Weiner S, Vaidyanathan TK. Effects of occlusal loading and thermocycling on the marginal gaps of light-polymerized and autopolymerized resin provisional crowns. J Prosthet Dent. 1999 Aug; 82(2):161-6.

How to cite this article: Choudhary M. Comparative evaluation of marginal fit of three provisional restorative materials in different mediums: an in-vitro study. International Journal of Research and Review. 2021; 8(3): 914. 